\title{
Role Of Point Of Care Ultrasound In The Diagnosis Of Retinal Detachment In The Emergency Department
}

This article was published in the following Dove Press journal: Open Access Emergency Medicine

\begin{abstract}
Ocular complaints represent up to $3 \%$ of all emergency department (ED) visits. These presentations can result in permanent vision loss if not promptly diagnosed and treated. Recently, point of care ultrasound (POCUS) has been investigated for the evaluation of ocular pathology in the ED. Multiple studies have investigated the role that ultrasound may play in the early diagnosis of these patients. Ocular ultrasound can be used to visualize the structures of the eye and evaluate for pathology such as retinal detachment, vitreous hemorrhage and vitreous detachment. This comprehensive review aims to present current evidence for the efficacy and reliability of ED ocular ultrasound use in the detection of retinal detachment and additional ocular pathologies.
\end{abstract}

Keywords: ocular ultrasound, retinal detachment, point of care ultrasound

\section{Introduction}

Ophthalmologic complaints make up approximately $3 \%$ of all emergency department (ED) visits. ${ }^{1}$ Several of these encounters are for acute eye pain, blurry vision, or visual impairment, with a differential diagnosis that includes a number of visionthreatening conditions requiring immediate and accurate assessment. ${ }^{2,3}$ Of this cohort, retinal detachment (RD) is a condition with a prevalence as high as $3.6 \%{ }^{4}$ Additionally, $\mathrm{RD}$ is considered a true ophthalmologic emergency and may require immediate surgical intervention to prevent irreversible vision loss. ${ }^{5,6}$ A thorough history and physical examination including biomicroscopy with slit lamp and measurement of intraocular pressures is often needed. Proper assessment of a patient with presumed retinal detachment requires a clear view to the posterior segment of the eye. ${ }^{7,8}$ Unfortunately, good visualization of the retina often involves specialized equipment, pupillary dilatation and expertise that may not readily accessible in the majority of EDs. ${ }^{9,10}$ Thus, history and physical examination alone may not be sufficient to make a formal diagnosis in an emergency room setting. ${ }^{11}$

With the advent of ocular ultrasound, there have been promising results for emergency medicine physicians to accurately identify ocular pathologies significantly reducing the burden of visual complaints in the ED. ${ }^{7,12-14}$ The efficiency, portability, and non-invasive characteristics of point-of-care ultrasound (POCUS) make it a valuable addition in the evaluation of patients with ophthalmic conditions. ${ }^{13}$ The use of POCUS for ocular pathology has greatly expanded in the past few years. ${ }^{13,15}$ The diagnostic capabilities of ocular ultrasound include an extensive list
Correspondence: Shadi Lahham University of California, Irvine, Department of Emergency Medicine, 333

The City Boulevard West, Suite 640,

Orange, CA 92868, USA

$\mathrm{Tel}+1$ 7|4-456-5922

Fax + I 7|4-456-37|4

Email slahham@uci.edu 
of ocular diseases including retinal detachment (RD), vitreous hemorrhage (VH), posterior vitreous detachment (PVD), intraocular foreign bodies, and increased intracranial pressure. ${ }^{7-9,13,16-18}$ Ultrasonography can be especially important when tools in the emergency room such as the direct ophthalmoscopy and tonometry are insufficient to make an assessment. ${ }^{12}$ Consequently, physicians have relied on ophthalmologic consultation to perform indirect ophthalmoscopy to form an appropriate evaluation. ${ }^{12}$ Furthermore, even with trained ophthalmologists, ultrasound becomes the only means of visualization of the posterior segment when the view through the anterior segment is opacified. ${ }^{16-18}$ Thus, ocular ultrasound has become an integral part of diagnosing posterior segment abnormalities.

While ocular ultrasonography has been used for decades by ophthalmologists, there has been emerging evidence behind the precision of identifying RDs with POCUS in the ED including a number of retrospective and prospective studies. ${ }^{14,18,19}$ Furthermore, a recent large-scale, prospective, multiple center study has demonstrated high sensitivity (96.9\%) and moderately specificity (88.1\%) in detecting retinal detachments. ${ }^{12}$ In patients presenting to the ED with ocular symptoms, POCUS can potentially aid in making the diagnosis and facilitate prompt ophthalmologic consultation. The purpose of this review is to evaluate the current literature on POCUS for the diagnosis of ocular pathology in the ED.

\section{Sonographic Physics And Anatomy}

Ocular ultrasound works by utilizing sound wave technology at high frequencies. These sound waves propagate through tissue and vibrate at different frequencies. Sound waves are amplified and create a pattern that can be interpreted at bedside. The visualization of ocular structures by sonogram is easily achieved due to its acoustically empty anterior chamber and vitreous cavity. The superficial location of the eye coupled with its fluid filled state create an ideal situation for ultrasound. Normal anatomy of the eye, as seen sonographically, consists of a hyperechoic lens, a clear posterior chamber, and a smooth retina. In a clear depiction, the optic nerve should lay flush against without protruding into the posterior chamber. With relation to the optic disc, fovea and macula regions of the eye are seen laterally. ${ }^{7}$

When using ultrasound to evaluate the eyes and orbits, several common tips and techniques may be incorporated. First, it is important to utilize a generous amount of lubricant in order to reduce signal loss between the probe and the tissue-air interface. Insufficient lubricant or ultrasound gel may lead to degraded image quality. Next, due to the superficial nature of the eye, a high-frequency linear probe is used to perform ocular ultrasound. The range for most ocular ultrasound setting is between 5 and $10 \mathrm{MHz} .^{7}$ However, due to the sensitivity of ocular tissue, the Food and Drug Administration (FDA) has strict requirements on both thermal index (TI) and mechanical index (MI) with limits less than 1.0 and 0.23 , respectively. $^{20,21}$ The thermal index is the ratio of the device acoustic power to power required to increase the temperature by $1{ }^{\circ} \mathrm{C}$ and the mechanical index is the estimate of the maximum amplitude of the pressure pulse in tissue and is an indicator of the likelihood of adverse mechanical (ie, non-thermal) bio-effect called cavitation. Because of the increased demand for ocular ultrasound, many ultrasound manufacturers include an "ocular" or "ophthalmologic" or "eye" software setting which automatically sets the TI and MI under the allowable limits.

\section{Sonographic Techniques}

Ocular ultrasound is typically performed in an upright or supine patient position. Ultrasound gel is placed on a closed eyelid and a linear ultrasound transducer is placed over the gel. Generally, two axes are used to visualize the eye and orbit. This includes transverse and longitudinal views. While each approach provides valuable information, the transverse is most commonly used in order to identify pathology. When performed correctly, the transverse view can help accurately measure optic nerve sheath diameter which may assist in detecting elevated intracranial pressure. $^{22}$ The longitudinal approach may assist in visualizing the contents of the eye, particularly in the posterior chamber. Typically, a static examination and kinetic echography are performed to thoroughly evaluate the globe. During a static examination, the patient holds the eye still and the sonographer fans through the orbit evaluating for pathology. During the kinetic echography, the sonographer holds the probe still and the patient is instructed to look left and right. ${ }^{7}$ This allows the sonographer to evaluate for the presence or absence of continued movement after the patient stops eye movement.

\section{Retinal Detachment And Other Applications}

Ocular sonography in the ED has shown promising results in the accuracy of detecting retinal detachment compared to other intraocular pathologies. ${ }^{12}$ Sonography is used as a 
helpful adjunct to the clinical assessment of RD and can provide results relatively quickly. Presenting symptoms often include acute onset of significant photopsias, persistent new floaters, flashes of light, and/or a constant, fixed visual field loss. While posterior segment abnormalities are challenging to diagnose in an emergency room setting with history, fundoscopy, and visual field testing alone, there are clear and distinguishable features on ocular ultrasound that differentiate retinal detachment to a non-pathological eye. Thus, the use of ocular ultrasound provides significant information to aid in the diagnosis of RD.

In a normal eye, the vitreous cavity appears as a circular hypoechoic structure with a hyperechoic retina that is indistinguishable from the underlying hyperechoic choroid (Figure 1). In the setting of a retinal detachment, a portion of the neurosensory retina is no longer contiguous with the choroid. Thus, the detached retina appears as a distinct hyperechoic line anterior to the choroid layer. In between the underlying choroid and the detached retina, there is a hypoechoic band of vitreous humor that has leaked to separate these two structures. Specifically, on POCUS, a RD is identified by the presence of a bright, echogenic membrane tethered to the optic disc (posteriorly) and the ora serrata (anteriorly), but separate from the choroid (Figure 2).

Once a retinal detachment has been identified, it is important to determine whether the macular region of the retina is attached (mac-on) versus separated (mac-off) from the choroid as the location of detachment will determine the urgency of treatment. Specifically, the macula

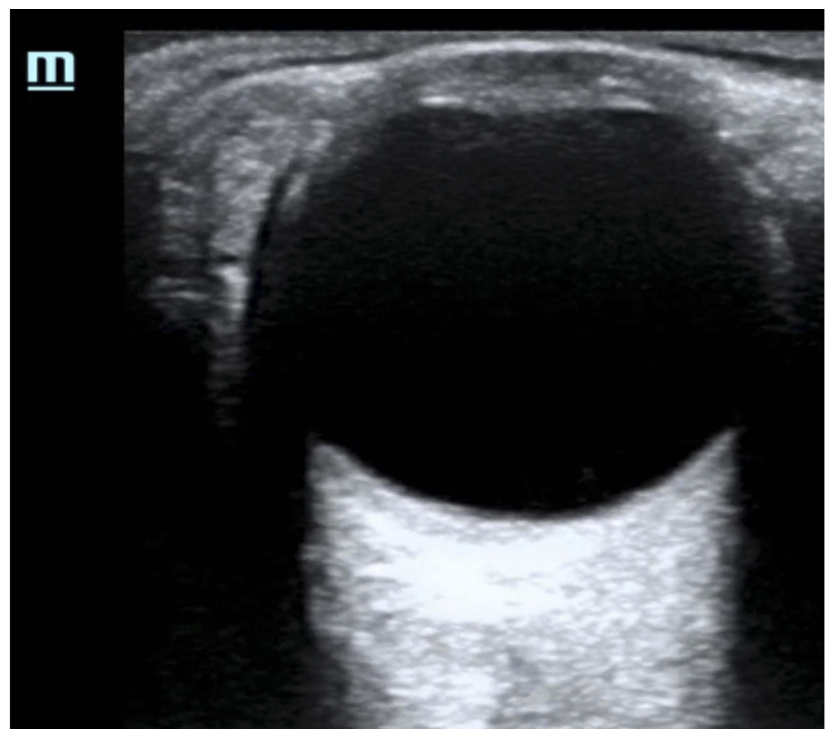

Figure I Point of care ultrasound image of normal eye.

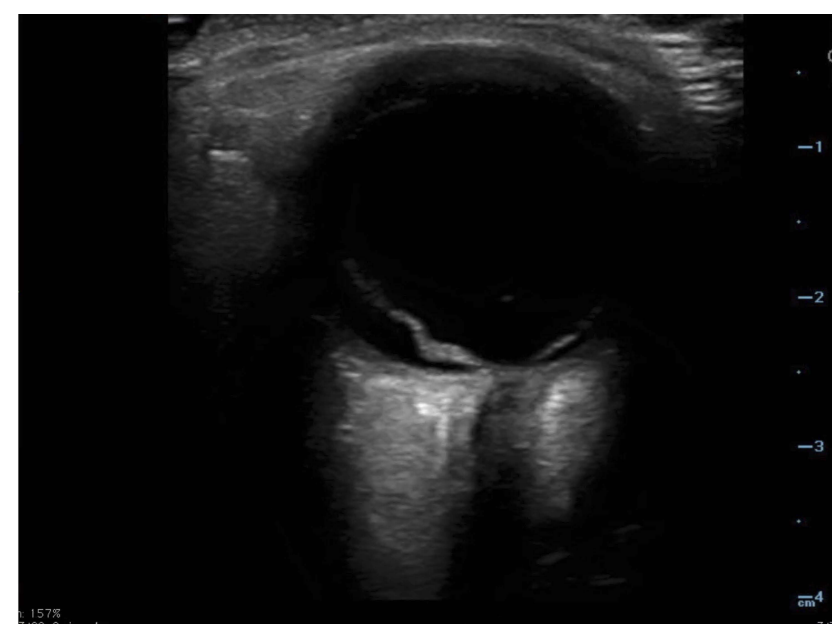

Figure 2 Point of care ultrasound image of retinal detachment.

lies lateral to the optic nerve and the posterior chamber in this region needs to be closely monitored to distinguish a mac-on from a mac-off detachment. The integrity of the retina at the macular region is essential for central vision and high-acuity vision. Consequently, in the case of a macon detachment where only peripheral retina has detached, emergent treatment is warranted in order to prevent the detachment to move centrally and affect the macula, which could result in irreversible vision loss and significantly impair central vision. ${ }^{7}$ In contrast to a mac-off detachment with significantly worse visual prognosis, there is no difference in visual outcomes if treatment was initiated within $24 \mathrm{hrs}$ or if treatment was delayed within 7 to 10 days. ${ }^{16}$ Treatment options for retinal detachment include surgical repair via pneumatic retinopexy, vitrectomy, or scleral buckle. $^{16}$

While features of RD are easily distinguished from a normal eye on POCUS, there are other ocular pathologies that may appear similar on POCUS. Previous studies have compared EPs ability to evaluate and distinguish both RD and other ocular pathology. ${ }^{12,15}$ Bedside ocular sonography has been incorporated in the detection of additional ocular pathology such as vitreous hemorrhage, vitreous detachment, ocular infections, foreign bodies, retrobulbar hematoma, and ocular vascular pathology. ${ }^{23}$ A study on the use of ocular ultrasound for the evaluation of retinal detachment by Shinar et al mandated a lecture on ocular sonography for EPs which emphasized different findings of three intraocular diseases including retinal detachment, vitreous hemorrhage, and vitreous detachment. ${ }^{15}$ A vitreous hemorrhage is seen as mobile, fluid collection of hyperechoic dots with variable 
echogenicity disconnected from the retina. Ultimately a vitreous hemorrhage ultrasound may simply demonstrate increased vitreous echogenicity or hyperechoic clots in the vitreous depending on the severity of the bleeding (Figure 3). In contrast, a posterior vitreous detachment shows a similar "swaying seaweed" of a hyperechoic strand, but is detached from the retina ${ }^{24}$ (Figure 4). It is important to note that multiple conditions can co-exist and often a vitreous hemorrhage can be a precursor to a retinal detachment.

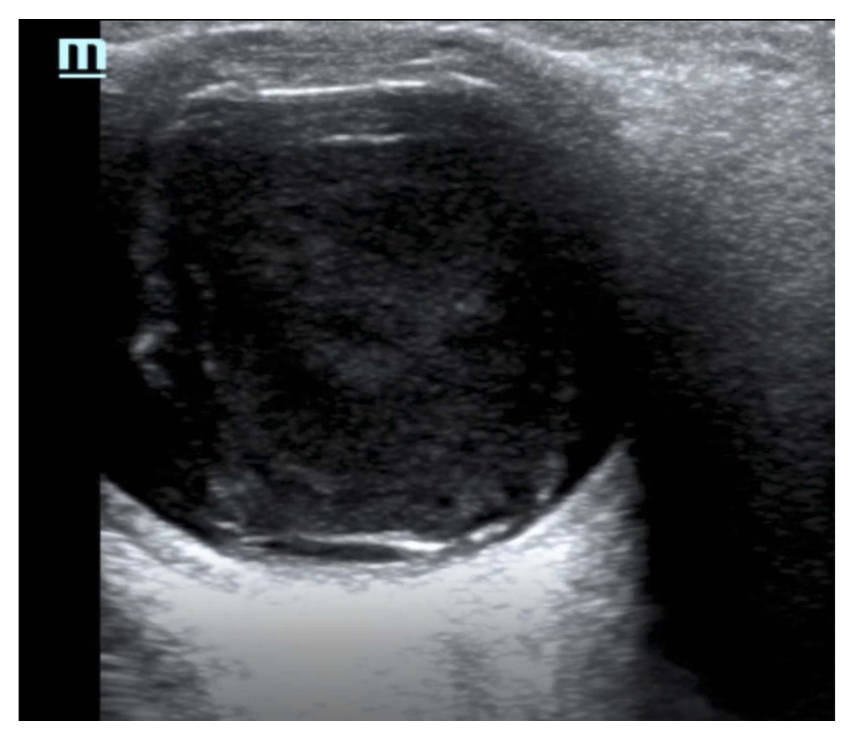

Figure 3 Point of care ultrasound image of vitreous hemorrhage.

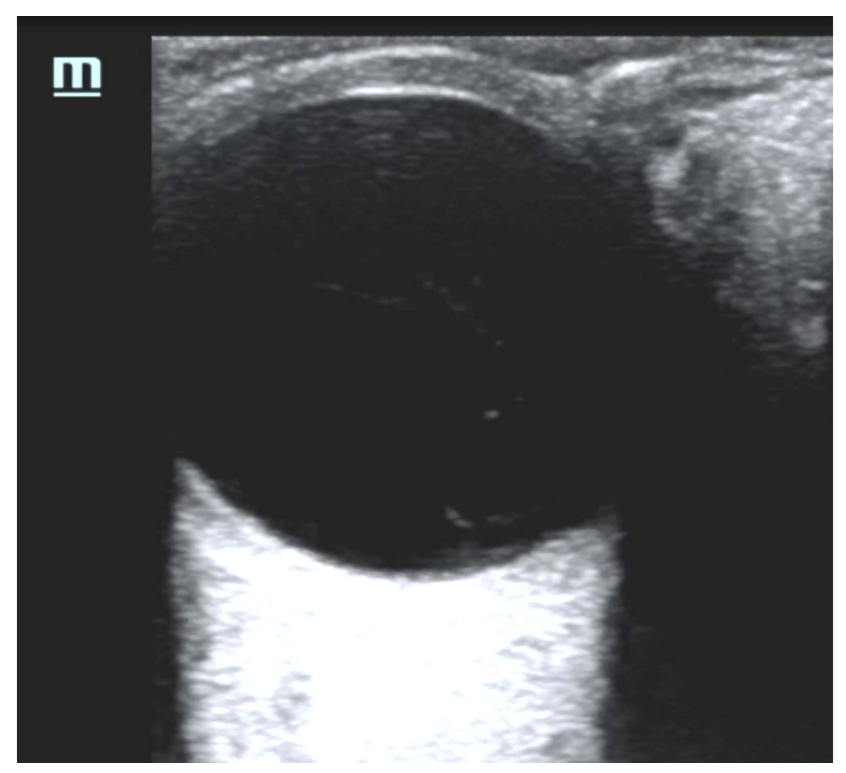

Figure 4 Point of care ultrasound image of vitreous detachment.

\section{Pitfalls And Limitations}

While several studies have demonstrated the ability for EPs to identify ocular pathology using POCUS, adequate training is required to obtain this skill. ${ }^{14}$ The amount of training required for proficiency is not yet known although several machine settings can be modified to help avoid pitfalls. When performing an ocular POCUS, depth and gain can be easily adjusted. It is important to avoid over-gaining an image when scanning the eye. Over-gaining the image can result in false positives as posterior vitreous humor becomes hyperechoic creating the illusion of a $\mathrm{RD}$ or $\mathrm{VH}^{25}$ As with ultrasonography of other anatomic structures, the strength of the signal can give more information on the pathology. While defects visualized using low or normal gain can be associated with RDs, adjusting gain is subject to user error. When testing kinetic movements under ultrasound, the ultrasonographer should ensure that the gain setting is not adjusted too quickly. Additionally, when differentiating pathology, it is important to note that posterior vitreous detachments and vitreous hemorrhages are not tethered to the retina, and adjusting the gain slowly will allow the ultrasonographer to rule out other pathologies. ${ }^{15}$ As mentioned earlier, ultrasound at high intensity has been shown to produce significant biologic effects through cavitation and thermal mechanisms..$^{20,21}$ To address this issue, specific ophthalmologic settings can be used to decrease levels of mechanical energy.

Another pitfall to avoid is related to the differentiation between mac-on and mac-off retinal detachments. The ophthalmologic treatment for these patients will differ as mac-on retinal detachments are treated more emergently. To date, there have been no large-scale studies that have evaluated patients using POCUS to differentiate between these different types of retinal detachments. One of the limitations acknowledged by Lahham et al was that sonographers were not asked to distinguish between mac-on and mac-off retinal detachments. ${ }^{12}$ Furthermore, patients with traumatic RDs were excluded from that study.

It is important to note that although ocular POCUS can help the EP distinguish between different types of ocular pathology, is not a substitute for an ophthalmology consultation. Rather, it should aid the evaluation of these patients. In cases where patients have concerning history or physical examinations with unremarkable POCUS, additional imaging or Ophthalmology consultation should be strongly considered to rule out subtle pathology including retinal tear or trace vitreous hemorrhage. EP performed physical exam and history alone are insufficient to rule out ocular pathology. 


\section{Conclusion}

Based on previous literature, EPs with the appropriate training can detect RDs using POCUS in the emergency department. Additionally, POCUS can be useful in differentiating between different types of ocular pathology. POCUS is not meant to replace the role of the ophthalmologist for definitive diagnosis, but may serve as an adjunct to help evaluate patients in the ED with ocular symptoms.

\section{Acknowledgment}

The authors would like to acknowledge the UC Irvine Department of Emergency Medicine.

\section{Disclosure}

Dr Fox receives stock options from Sonosim for consulting. The authors report no other conflicts of interest in this work.

\section{References}

1. Blaivas M, Theodoro D, Sierzenski PR. A study of bedside ocular ultrasonography in the emergency department. Acad Emerg Med. 2002;9(8):791-799. doi:10.1197/aemj.9.8.791

2. Walker RA, Adhikari S. Chapter 236 p1517-1549. Eye emergencies. In: Tintinali JE, Kelen GD, Stapczynski JS, editors. Tintinali's Emergency Medicine: A Comprehensive Study Guide. 7th ed. New York: McGraw- Hill; 2011.

3. Jafari AK, Bozorgui S, Shahverdi N, et al. Different causes of referral to ophthalmology emergency room. J Emerg Trauma Shock. 2012;5 (1):16-22. doi:10.4103/0974-2700.93104

4. Alotaibi AG, Osman EA, Allam KH, et al. One month outcome of ocular related emergencies in a tertiary hospital in central Saudi Arabia. Saudi Med J. 2011;32:1256-1260.

5. Haimann MH, Burton TC, Brown CK. Epidemiology of retinal detachment. Arch Ophthalmol. 1982;100(2):289-292. doi:10.1001/ archopht.1982.01030030291012

6. Nagpal M, Chaudhary P, Wachasundar S, et al. Management of recurrent rhegmatogenous retinal detachment. Indian J Ophthalmol. 2018;66 (12):1763-1771. doi:10.4103/ijo.IJO 121218

7. Fox J, editor. Clinical Emergency Radiology. Cambridge: Cambridge University Press. 2008;I-Vi:324-330.

8. De La Hoz Polo M, Torramilans Lluís A, Pozuelo Segura O, et al. Ocular ultrasonography focused on the posterior eye segment: what radiologists should know. Insights Imaging. 2016;7(3):351-364. doi:10.1007/s13244-016-0471-z

9. Roque PJ, Hatch N, Barr L, et al. Bedside ocular ultrasound. Crit Care Clin. 2014;30(2):227-241. doi:10.1016/j.ccc.2013.10.007. Epub 2013 Dec 12. PubMed PMID: 24606775.

10. Tandon A, Khullar T, Bhatt S. Sonography in acute ocular pathology: a kaleidoscopic view. Emerg Radiol. 2019;26(2):241-248. doi:10.1007/s10140-018-1655-2. Epub 2018 Nov 16. Review. PubMed PMID: 30446852.
11. Vrablik ME, Snead GR, Minnigan HJ, et al. The diagnostic accuracy of bedside ocular ultrasonography for the diagnosis of retinal detachment: a systematic review and meta-analysis. Ann Emerg Med. 2015;65(2):199-203.e1. doi:10.1016/j.annemergmed.2014.02.020

12. Lahham S, Shniter I, Thompson M, et al. Point-of-care ultrasonography in the diagnosis of retinal detachment, vitreous hemorrhage, and vitreous detachment in the emergency department. JAMA Netw Open. 2019;2(4): e192162. doi:10.1001/jamanetworkopen.2019.2162. PMID: 30977855.

13. Baker N, Amini R, Situ-LaCasse EH, et al. Can emergency physicians accurately distinguish retinal detachment from posterior vitreous detachment with point-of-care ocular ultrasound? Am J Emerg Med. 2018;36(5):774-776. doi:10.1016/j.ajem.2017.10.010. Epub 2017 Oct 13. PubMed PMID: 29042095.

14. Jacobsen B, Lahham S, Lahham S, et al. Retrospective review of ocular point-of-care ultrasound for detection of retinal detachment. West J Emerg Med. 2016;17(2):196-200. doi:10.5811/westjem.2015.12.28711. Epub 2016 Mar 2. PubMed PMID: 26973752; PubMed Central PMCID: PMC4786246.

15. Shinar Z, Chan L, Orlinsky M. Use of ocular ultrasound for the evaluation of retinal detachment. J Emerg Med. 2011;40(1):53-57. doi:10.1016/j.jemermed.2009.06.001

16. Gerstenblith A, Rabinowitz M. The Wills Eye Manual: Office and Emergency Room Diagnosis and Treatment of Eye Disease. Philadelphia:Lippincott, 1994. Philadelphia (PA): Wolters Kluwer Publishing; 2012.

17. Fielding JA. Ocular ultrasound. Clin Radiol. 1996;51(8):533-544. doi:10.1016/S0009-9260(96)80131-0

18. Blumenkranz MS, Byrne SF. Standardized echography (ultrasonography) for the detection and characterization of retinal detachment. Ophthalmology. 1982;89(7):821-831. doi:10.1016/S0161-6420(82) 34716-8. PubMed PMID: 7122054.

19. Kim DJ, Francispragasam M, Docherty G, et al. Test characteristics of point-of-care ultrasound for the diagnosis of retinal detachment in the emergency department. Acad Emerg Med. 2019;26(1):16-22. doi:10.1111/acem.13454. Epub 2018 Dec 17. PubMed PMID 29774966.

20. Food and Drug Administration. Guidance for Industry and FDA Staff Information for Manufacturers Seeking Marketing Clearance of Diagnostic Ultrasound Systems and Transducers. Maryland: Silver Springs; 2008 Sep 9.

21. Barnett SB. World Federation for Ultrasound in Medicine and Biology (WFUMB) Symposium on safety of ultrasound in medicine: conclusions and recommendations on thermal and non-thermal mechanisms for biological effects of ultrasound. Ultrasound Med Biol. 1998;24:1-55.

22. Blaivas M, Theodoro D, Sierzenski P. Elevated intracranial pressure detected by bedside emergency ultrasonography of the optic nerve sheath. Acad Emer Med. 2003;10:376-381. doi:10.1197/aemj.10.4.376

23. Kilker BA, Holst JM, Hoffmann B. Bedside ocular ultrasound in the emergency department. Eur J Emerg Med. 2014;21(4):246-253. doi:10.1097/MEJ.0000000000000070

24. Schott ML, Pierog JE, Williams SR. Pitfalls in the use of ocular ultrasound for evaluation of acute vision loss. J Emerg Med. 2013;44:1136-1139. doi:10.1016/j.jemermed.2012.11.079

25. Waldron RG. Contact B-Scan Ultrasonography. Emory Eye Center; 2003:1-9. 


\section{Publish your work in this journal}

The Open Access Emergency Medicine is an international, peerreviewed, open access journal publishing original research, reports, editorials, reviews and commentaries on all aspects of emergency medicine. The manuscript management system is completely online

Submit your manuscript here: https://www.dovepress.com/open-access-emergency-medicine-journal and includes a very quick and fair peer-review system, which is all easy to use. Visit http://www.dovepress.com/testimonials.php to read real quotes from published authors. 